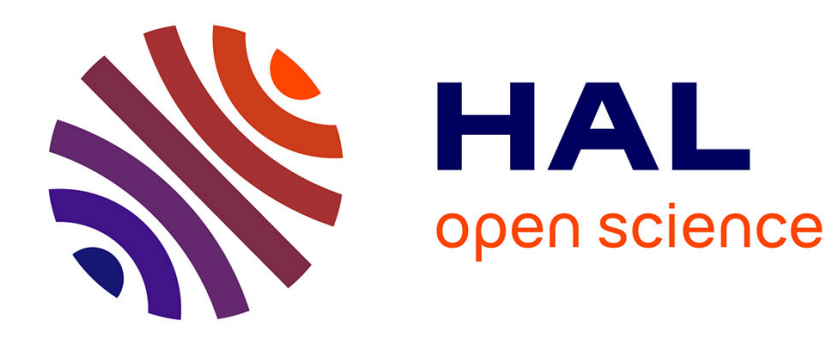

\title{
Optimal Energy Decay Rate in a Damped Rayleigh Beam.
}

\author{
Bopeng Rao
}

\section{To cite this version:}

Bopeng Rao. Optimal Energy Decay Rate in a Damped Rayleigh Beam.. 1997. hal-00129564

\section{HAL Id: hal-00129564 https://hal.science/hal-00129564}

Preprint submitted on 8 Feb 2007

HAL is a multi-disciplinary open access archive for the deposit and dissemination of scientific research documents, whether they are published or not. The documents may come from teaching and research institutions in France or abroad, or from public or private research centers.
L'archive ouverte pluridisciplinaire HAL, est destinée au dépôt et à la diffusion de documents scientifiques de niveau recherche, publiés ou non, émanant des établissements d'enseignement et de recherche français ou étrangers, des laboratoires publics ou privés. 


\title{
Optimal Energy Decay Rate in a Damped Rayleigh Beam
}

\author{
Bopeng Rao \\ Institut de Recherche Mathématique Avancée \\ Université de Louis Pasteur de Strasbourg \\ 7 Rue René-Descartes, 67084 Strasbourg Cedex, France \\ E-mail : rao@math.u-strasbg.fr
}

Abstract. - We consider a clamped Rayleigh beam subject to a positive viscous damping. Using an explicit approximation, we first give the asymptotic expansion of eigenvalues and eigenfunctions of the underlying system. We next identify the optimal energy decay rate of the system with the supremum of the real part of the spectrum of the infinitesimal generator of the associated semigroup.

\section{Introduction}

We consider a clamped Rayleigh beam in the presence of viscous damping $2\left(a y_{t x}\right)_{x}$ :

$$
\left\{\begin{array}{l}
y_{t t}-\gamma^{2} y_{x x t t}+y_{x x x x}-2\left(a y_{t x}\right)_{x}=0, \quad 0<x<1, \quad t>0 \\
y(0, t)=y_{x}(0, t)=y(1, t)=y_{x}(1, t)=0, \quad t>0, \\
y(x, 0)=y_{0}(x), \quad y_{t}(x, 0)=y_{1}(x)
\end{array}\right.
$$

where $\gamma^{2}>0$ is the coefficient of moment of inertia, and where the ponential $a \in L^{\infty}(0,1)$ is a positive function. For more details concerning the modelling of the system, we refer to Russell [15].

Let $H=H_{0}^{2}(0,1) \times H_{0}^{1}(0,1)$ endowed with the inner product :

$$
\langle(y, z),(f, g)\rangle_{H}=\int_{0}^{1}\left(y_{x x} \bar{f}_{x x}+z \bar{g}+\gamma^{2} z_{x} \bar{g}_{x}\right) d x .
$$

We define the linear unbounded operator $A$ by

$$
A=\left(\begin{array}{cc}
0 & I \\
-G_{2} \partial_{x x x x} & 2 G_{2} \partial_{x}\left(a \partial_{x}\right)
\end{array}\right), \quad D(A)=\left(H^{3}(0,1) \cap H_{0}^{2}(0,1)\right) \times H_{0}^{2}(0,1) .
$$

1991 Mathematics Subject Classification. Primary 35P20; Secondary 93D15. 
where

$$
G_{2}:=\left(I-\gamma^{2} \partial_{x x}\right)^{-1}
$$

is an isomorphism from $H^{-1}(0,1)$ onto $H_{0}^{1}(0,1)$.

Setting $u=\left(y, y_{t}\right)$, we transform the equation (1.1) into an evolutionary equation

$$
u_{t}=A u, \quad u(0)=u_{0} \in H .
$$

Since $A$ is a bounded monotone perturbation of a skew adjoint operator (undamped), then it is easily seen that $A$ generates a $C_{0}$ semigroup $S(t)$ of contractions on the energy space $H$ (e.g. Pazy [13], Lagnese [10], Rao [14]). Moreover, if we assume that $a$ is strictly positive on a subinterval, then we can easily prove that there exist positive constants $M, \omega$ such that the following exponential decay rate holds

$$
\|S(t)\| \leq M \exp (-\omega t), \quad \forall t \geq 0 .
$$

But our goal is to find the optimal energy decay rate. More precisely, denoting by $\omega(a)$ the supremum of $\omega$ satisfying (1.6), and by $\mu(a)$ the supremum of the real part of the eigenvalues of $A$, we have obviously $\omega(a) \leq \mu(a)$. We will establish the reverse inequality for the coefficient $a \in W^{3,1}(0,1)$ by proving that the system of eigenvectors of the operator $A$ constitutes a Riesz basis in the energy space $H$. For this end, we will apply Bari's theorem which, by its constructive nature, requires very precise knowledge of the spectrum of $A$.

The present work is inspired deeply on that of Cox-Zuazua [6,7] on the wave equation with a nonnegative potential term. The shooting method based on an ansatz of Horn was applied to obtain the asymptotic expansion of eigenvalues of the system. But their shooting arguments do not appear to be easily generalized to the beam problems. In fact in order to adapt the shooting method to the beam equation, we have to establish an adequat a priori estimate which is not evident even for the wave equation (see Freitas-Zuazua [8]). In order to obtain the asymptotic expansion of eigenvalues and eigenvectors of the operator $A$, we propose an explicit approximation the characteristic determinant of the underlying system. Unlike the usual asymptotic expansion method, our approach is successive and based on the high frequencies of the system. This point seems to be new.

The second part of this work consists in construcing a Riesz basis which is quadratically close to the system of eigenvectors of $A$. Consequently, we identify the optimal energy decay rate with the supremum of the real part of $A$.

There are many results on the spectrum of the beam equations (see e.g. Chen et al. [2, 3], Russell [15]). But most of them are obtained by using the explicit characteristic equation of the underlying system. In the case of variable coefficient the main difficulty is that the characteristic equation is not explicit. Here we will adopt the classical shooting method which has been systematically studied by Birkoff-Langer [1]. Interesting results for a damped string equation were obtained in Chen et al. [4] and Cox-Zuazua [6, 7]. In particular, an average property on the spectrum of Euler-Bernoulli beam equation was conjectured in Chen et al. [4]. 


\section{Lower frequencies}

In this section, we assume that the coefficient function $a$ satisfies

$$
0<\alpha \leq a(x) \leq \beta<+\infty, \quad 0 \leq x \leq 1 .
$$

Proposition 2.1. - The complex eigenvalues of $A$ are contained in

$$
\left\{|\lambda|^{2} \geq \frac{\pi^{4}}{1+\gamma^{2} \pi^{2}}, \quad-\frac{\beta}{\gamma^{2}} \leq \operatorname{Re} \lambda \leq-\frac{\alpha \pi^{2}}{1+\gamma^{2} \pi^{2}}\right\} .
$$

A necessary condition for existence of real eigenvalues is

$$
\beta>\frac{\gamma^{2} \pi^{2}}{\sqrt{1+\gamma^{2} \pi^{2}}}
$$

In that case, we have

$$
-\frac{\beta}{\gamma^{2}}-\sqrt{\frac{\beta^{2}}{\gamma^{4}}-\frac{\pi^{4}}{1+\gamma^{2} \pi^{2}}} \leq \lambda_{n} \leq-\frac{\beta}{\gamma^{2}}+\sqrt{\frac{\beta^{2}}{\gamma^{4}}-\frac{\pi^{4}}{1+\gamma^{2} \pi^{2}}} .
$$

Proof. - Let $\lambda_{n}$ be an eigenvalue and $\phi_{n}=\left(y_{n}, z_{n}\right)$ be an associated eigenfunction. Then we have

$$
\left\{\begin{array}{l}
z_{n}=\lambda_{n} y_{n} \\
-y_{n x x x x}+2\left(a z_{n x}\right)_{x}=\lambda_{n}\left(z_{n}-\gamma^{2} z_{n x x}\right) .
\end{array}\right.
$$

This gives

$$
\left\{\begin{array}{l}
\lambda_{n}^{2} y_{n}-\gamma^{2} \lambda_{n}^{2} y_{n x x}+y_{n x x x x}-2 \lambda_{n}\left(a y_{n x}\right)_{x}=0 \\
y_{n}(0)=y_{n x}(0)=y_{n}(1)=y_{n x}(1)=0 .
\end{array}\right.
$$

Assume that

$$
\int_{0}^{1}\left(\left|y_{n}\right|^{2}+\gamma^{2}\left|y_{n x}\right|^{2}\right) d x=1
$$

Then using Poincaré's inequality, we deduce that

$$
\frac{\pi^{2}}{1+\gamma^{2} \pi^{2}} \leq \int_{0}^{1}\left|y_{n x}\right|^{2} d x<\frac{1}{\gamma^{2}}
$$

Now multiplying the equation (2.5) by $y_{n}$ and integrating by parts we obtain that

$$
\lambda_{n}^{2}+2 \lambda_{n} \int_{0}^{1} a\left|y_{n x}\right|^{2} d x+\int_{0}^{1}\left|y_{x x}\right|^{2} d x=0 .
$$


It follows that

$$
\lambda_{n}=-\int_{0}^{1} a\left|y_{n x}\right|^{2} d x \pm \sqrt{\left(\int_{0}^{1} a\left|y_{n x}\right|^{2} d x\right)^{2}-\int_{0}^{1}\left|y_{n x x}\right|^{2} d x} .
$$

If $\lambda_{n}$ is a complex eigenvalue, then we have

$$
\operatorname{Re} \lambda_{n}=-\int_{0}^{1} a\left|y_{n x}\right|^{2} d x, \quad \operatorname{Im} \lambda_{n}=\sqrt{\int_{0}^{1}\left|y_{n x x}\right|^{2} d x-\left(\int_{0}^{1} a\left|y_{n x}\right|^{2} d x\right)^{2}} .
$$

Using (2.7) into (2.10) gives the estimate (2.2).

If $\lambda_{n}$ is a real eigenvalue, then using (2.7) and Poincaré's inequality, we get

$$
\frac{\beta^{2}}{\gamma^{4}}>\left(\int_{0}^{1} a\left|y_{n x}\right|^{2} d x\right)^{2} \geq \int_{0}^{1}\left|y_{n x x}\right|^{2} d x \geq \frac{\pi^{4}}{1+\gamma^{2} \pi^{2}}
$$

This gives the condition (2.3).

Finally since the function $(s, t) \rightarrow-s+\sqrt{s^{2}-t}$ is increasing with respect to $s$ and decreasing with respect to $t$ (respectively the function $(s, t) \rightarrow-s-\sqrt{s^{2}-t}$ is decreasing with respect to $s$ and increasing with respect to $t$ ), then inserting (2.11) into (2.9), we obtain the estimate (2.3). This achieves the proof.

Now following an idea of Cox and Zuazua [6], we consider the parametrized self-adjoint eigenvalue problem :

$$
\left\{\begin{array}{l}
\lambda^{2} \psi_{n}-\gamma^{2} \lambda^{2} \psi_{n x x}+\psi_{n x x x x}-2 \nu_{n}\left(a \psi_{n x}\right)_{x}=0 \\
\psi_{n}(0)=\psi_{n x}(0)=\psi_{n}(1)=\psi_{n x}(1)=0
\end{array}\right.
$$

where the eigenvalues $\nu_{n}$ is ordered

$$
0>\nu_{1}(\lambda) \geq \nu_{2}(\lambda) \geq \cdots \geq \nu_{n}(\lambda) \rightarrow-\infty
$$

We observe that $\lambda$ is an eigenvalue of the operator $A$ if and only if $\lambda$ is a fixed point of the application $\lambda \rightarrow \nu_{n}(\lambda)$ for some integer $n$.

Let $\mu_{n}>0$ be the $n$th eigenvalue of the problem

$$
\left\{\begin{array}{l}
\phi_{x x x x}+\mu \phi_{x x}=0 \\
\phi(0)=\phi_{x}(0)=\phi(1)=\phi_{x}(1)=0
\end{array}\right.
$$

Then a straightforward computation gives that

$$
(n-1)^{2} \pi^{2}<\mu_{n} \leq n^{2} \pi^{2} .
$$

From the minmax characterization

$$
\nu_{n}(\lambda)=-\min _{E_{n}} \max _{\psi \in E_{n}} \frac{\int_{0}^{1} \psi_{x x}^{2} d x+\gamma^{2} \lambda^{2} \int_{0}^{1} \psi_{x}^{2} d x+\lambda^{2} \int_{0}^{1} \psi^{2} d x}{2 \int_{0}^{1} a \psi_{x}^{2} d x} .
$$


we deduce the following estimate

$$
-\frac{\left(\gamma^{2}+\frac{1}{\pi^{2}}\right) \lambda^{2}+n^{2} \pi^{2}}{2 \alpha} \leq \nu_{n}(\lambda) \leq-\frac{\gamma^{2} \lambda^{2}+(n-1)^{2} \pi^{2}}{2 \beta} .
$$

TheOrem 2.2. - Assume that

$$
\alpha^{2}>n^{2} \pi^{2}\left(\gamma^{2}+\frac{1}{\pi^{2}}\right)
$$

Then $A$ has at least $2 n$ real eigenvalues.

Proof. - Denoting by $\alpha_{k}^{ \pm}$the roots of the equation

$$
\left(\gamma^{2}+\frac{1}{\pi^{2}}\right) \lambda^{2}+2 \alpha \lambda+k^{2} \pi^{2}=0
$$

and by $\beta_{k}^{ \pm}$the roots of the equation

$$
\gamma^{2} \lambda^{2}+2 \beta \lambda+(k-1)^{2} \pi^{2}=0
$$

then under the condition (2.18), we see that the roots $\alpha_{k}^{ \pm}, \beta_{k}^{ \pm}$are negative numbers.

On the other hand, from (2.17) we deduce that

$$
-\frac{\left(\gamma^{2}+\frac{1}{\pi^{2}}\right) \lambda^{2}+2 \alpha \lambda+k^{2} \pi^{2}}{2 \alpha} \leq \nu_{n}(\lambda)-\lambda \leq-\frac{\gamma^{2} \lambda^{2}+2 \beta \lambda+(k-1)^{2} \pi^{2}}{2 \beta} .
$$

This implies in particular that $\nu_{k}(\lambda)$ admits a fixed point $\lambda_{k}^{-} \in\left[\beta_{k}^{-}, \alpha_{k}^{-}\right]$and a fixed point $\lambda_{k}^{+} \in\left[\alpha_{k}^{+}, \beta_{k}^{+}\right]$. More specifically, we have

$$
\begin{aligned}
& \frac{-\beta-\sqrt{\beta^{2}-(k-1)^{2} \pi^{2} \gamma^{2}}}{2 \gamma^{2}} \leq \lambda_{k}^{-} \leq \frac{-\alpha-\sqrt{\alpha^{2}-k^{2} \pi^{2}\left(\gamma^{2}+\frac{1}{\pi^{2}}\right)}}{2 \gamma^{2}} . \\
& \frac{-\alpha+\sqrt{\alpha^{2}-k^{2} \pi^{2}\left(\gamma^{2}+\frac{1}{\pi^{2}}\right)}}{2 \gamma^{2}} \leq \lambda_{k}^{+} \leq \frac{-\beta+\sqrt{\beta^{2}-(k-1)^{2} \pi^{2} \gamma^{2}}}{2 \gamma^{2}}
\end{aligned}
$$

TheOREm 2.3. - A has a real eigenvalue if and only if there exists a nontrivial function $\psi \in H_{0}^{2}(0,1)$ such that

$$
F(\psi)=\left(\int_{0}^{1} a \psi_{x}^{2} d x\right)^{2}-\int_{0}^{1} \psi_{x x}^{2} d x \int_{0}^{1}\left(\psi^{2}+\gamma^{2} \psi_{x}^{2}\right) d x \geq 0
$$

Proof. - The necessary condition follows from (2.9). For the sufficient condition using (2.16) we have

$$
\frac{\gamma^{2} \lambda^{2}+2 \beta \lambda}{2 \beta} \leq \lambda-\nu_{1}(\lambda) \leq \frac{\int_{0}^{1} \psi_{x x}^{2} d x+2 \lambda \int_{0}^{1} a \psi_{x}^{2} d x+\lambda^{2} \int_{0}^{1}\left(\psi^{2}+\gamma^{2} \psi_{x}^{2}\right) d x}{2 \int_{0}^{1} a \psi_{x}^{2} d x}
$$


Since the left-hand side of (2.22) go to $+\infty$ as $\lambda \rightarrow-\infty$, then $\nu_{1}(\lambda)$ admits a fixed point when there exists a nontrivial function $\psi \in H_{0}^{2}(0,1)$ such that the right-hand side of (2.22) is negative for some negative $\lambda$. This gives the condition (2.21). The proof is complete.

Theorem 2.4. - If A has a real eigenvalue, then $a_{0}=\int_{0}^{1} a(x) d x>\gamma$.

Proof. - We first recall a result of Cox and Zuazua [6].

$$
\left(\int_{0}^{1} a y^{2} d x\right)^{2} \leq a_{0}^{2} \int_{0}^{1} y_{x}^{2} d x \int_{0}^{1} y^{2} d x, \quad \forall y \in H_{0}^{1}(0,1) .
$$

Since there exists nontrivial $\psi \in H_{0}^{2}(0,1)$ satisfying (2.21), then using (2.23) we have

$$
\int_{0}^{1} \psi_{x x}^{2} d x \int_{0}^{1}\left(\psi^{2}+\gamma^{2} \psi_{x}^{2}\right) d x \leq\left(\int_{0}^{1} a \psi_{x}^{2} d x\right)^{2} \leq a_{0}^{2} \int_{0}^{1} \psi_{x x}^{2} d x \int_{0}^{1} \psi_{x}^{2} d x .
$$

This achieves the proof.

TheOrem 2.5. - If $a_{0} \leq \gamma$, then the spectrum of $A$ is contained in the sector

$$
\rho>0, \quad \frac{\pi}{2}<\theta<\arcsin \left(\frac{a_{0}}{\gamma}\right)+\frac{\pi}{2} .
$$

Proof. - Let $\lambda=\rho e^{i \theta} \in \sigma(A)$, we write $\theta=\frac{\pi}{2}+\theta_{0}$ with $0<\theta_{0}<\frac{\pi}{2}$. From (2.10), it follows that

$$
\frac{\sin ^{2} \theta_{0}}{\cos ^{2} \theta_{0}}=\frac{\int_{0}^{1} a y_{n x}^{2} d x}{\int_{0}^{1} y_{n x x}^{2} d x-\int_{0}^{1} a y_{n x}^{2} d x} .
$$

Using (2.23) and (2.7), it follows that

$$
0<\frac{\sin ^{2} \theta_{0}}{\cos ^{2} \theta_{0}} \leq \frac{a_{0}^{2} \int_{0}^{1} y_{n x}^{2} d x}{1-a_{0}^{2} \int_{0}^{1} y_{n x}^{2} d x}<\frac{a_{0}^{2}}{\gamma^{2}-a_{0}^{2}} .
$$

This implies that $0<\theta_{0}<\arcsin \left(\frac{a_{0}}{\gamma}\right)$. The proof is complete.

\section{High frequencies}

In this section, we will adapt the shooting method to the beam equation. To this end, we propose an explicit approximation of the eigenfunction of the problem (2.5).

Now we give the idea of the shooting method. Instead of the boundary value problem (2.5), we first consider the following initial value problems :

$$
\left\{\begin{array}{l}
\lambda^{2} y_{1}-\gamma^{2} \lambda^{2} y_{1 x x}+y_{1 x x x x}-2 \lambda\left(a y_{1 x}\right)_{x}=0, \\
y_{1}(0)=y_{1 x}(0)=y_{1 x x}(0)=0, \quad y_{1 x x x}(0)=1 .
\end{array}\right.
$$




$$
\left\{\begin{array}{l}
\lambda^{2} y_{2}-\gamma^{2} \lambda^{2} y_{2 x x}+y_{1 x x x x}-2 \lambda\left(a y_{2 x}\right)_{x}=0, \\
y_{2}(0)=y_{2 x}(0)=y_{2 x x x}(0)=0, \quad y_{2 x x}(0)=1 .
\end{array}\right.
$$

Since the coefficients, as well as the initial data, of the equation (3.1) and (3.2) are analytic functions of the complex variable $\lambda$, then by the classical theory of linear ordinary differential equation (e.g. Coddington-Levinson [5, p. 36 and 40], Naimak [12, p.13-14]) we know that the equations (3.1) and (3.2) have the unique solutions $y_{1}$ and $y_{2}$ which are analytic functions of the complex variable $\lambda$.

We next define

$$
Y(x, \lambda)=y_{1}(1, \lambda) y_{2}(x, \lambda)-y_{2}(1, \lambda) y_{1}(x, \lambda)
$$

Then we have

$$
\left\{\begin{array}{l}
\lambda^{2} Y-\gamma^{2} \lambda^{2} Y_{x x}+Y_{x x x x}-2 \lambda\left(a Y_{x}\right)_{x}=0, \\
Y(0, \lambda)=Y_{x}(0, \lambda)=Y(1, \lambda)=0 .
\end{array}\right.
$$

Obviously if $Y_{x}\left(1, \lambda_{n}\right)=0$, then $\lambda_{n}$ is an eigenvalue of the operator $A$. Inversely we have the following result.

TheOREm 3.1. - Let $\lambda_{n}$ be an eigenvalue of $A$, then $\lambda_{n}$ is a zero of the function $\lambda \rightarrow Y_{x}(1, \lambda)$. Moreover the algebraic multiplicity of $\lambda_{n}$ is the order of $\lambda_{n}$ as the zero of the function $\lambda \rightarrow Y_{x}(1, \lambda)$.

Proof. - Let $A\left(y_{n}, z_{n}\right)=\lambda_{n}\left(y_{n}, z_{n}\right)$. Then $z_{n}=\lambda_{n} y_{n}$, where $y_{n}$ satisfies the equation (2.5). Since $y_{n}(0)=y_{n x}(0)=0$, there exist constants $C_{1}, C_{2}$ such that

$$
y_{n}(x)=C_{1} y_{1}\left(x, \lambda_{n}\right)+C_{2} y_{2}\left(x, \lambda_{n}\right) .
$$

Since $y_{n}(1)=y_{n x}(1)=0$, it follows that

$$
\left\{\begin{array}{l}
C_{1} y_{1}\left(1, \lambda_{n}\right)+C_{2} y_{2}\left(1, \lambda_{n}\right)=0, \\
C_{1} y_{1 x}\left(1, \lambda_{n}\right)+C_{2} y_{2 x}\left(1, \lambda_{n}\right)=0 .
\end{array}\right.
$$

We observe that $Y_{x}\left(1, \lambda_{n}\right)$ is the determinant of the linear system (3.6). Therefore from the classical theory of linear differential operators (see Naimark [12, p.14]), we deduce that $\lambda_{n}$ is an eigenvalue of $A$ if and only if $\lambda_{n}$ is a zero of the analytic function $Y_{x}(1, \lambda)$. Moreover the algebraic multiplicity of $\lambda_{n}$ is the order of $\lambda_{n}$ as a zero of the function $Y_{x}(1, \lambda)$. The proof is complete.

We will prove later that the rank of the linear system (3.6) is of one for $|\lambda|$ sufficiently large. In that case the geometric multiplicity of $\lambda_{n}$ is also of one, and the associated eigenfunction is a scalar multiple of $Y\left(x, \lambda_{n}\right)$. Therefore it is nartural to expect that a good approximation of $Y(x, \lambda)$ will yield a good asymptotic expansion of eigenvalues and eigenvectors of the problem (2.5). 
In order to the solve the initial value problems (3.1)-(3.2), we propose an explicit approximation. For clarity, we divide the construction into several steps.

Step 1. Estimates of the solution $z_{1}$. Let $z_{1}$ be the solution of the undamped equation :

$$
\left\{\begin{array}{l}
\lambda^{2} z_{1}-\gamma^{2} \lambda^{2} z_{1 x x}+z_{1 x x x x}=0 \\
z_{1}(0)=z_{1 x}(0)=z_{1 x x}(0)=0, \quad z_{1 x x x}(0)=1
\end{array}\right.
$$

Then the solution $z_{1}$ is given explicitly by

$$
z_{1}=\frac{1}{\tau_{1}^{2}-\tau_{2}^{2}}\left(\frac{1}{\tau_{1}} \sinh \tau_{1} x-\frac{1}{\tau_{2}} \sinh \tau_{2} x\right)
$$

where the frequencies $\tau_{1,2}$ are given by

$$
\tau_{1,2}=\sqrt{\frac{\gamma^{2} \lambda^{2} \pm \sqrt{\gamma^{4} \lambda^{4}-4 \lambda^{2}}}{2}}
$$

Then a straightforward computation gives

$$
\tau_{1}=\gamma \lambda+O\left(\frac{1}{|\lambda|}\right), \quad \tau_{2}=\frac{1}{\gamma}+O\left(\frac{1}{|\lambda|^{2}}\right) .
$$

By virtue of Theorem 2.1, we know that the spectrum $\sigma(A)$ is contained in the strip $-2 \beta / \gamma^{2} \leq \operatorname{Re} \lambda \leq 0$, wherein the functions $\sinh \tau_{1} x, \sinh \tau_{2} x$ are uniformly bounded. Therefore we find easily

$$
\left|z_{1}\right| \leq \frac{C}{|\lambda|^{2}}, \quad\left|z_{1 x}\right| \leq \frac{C}{|\lambda|^{2}}, \quad\left|z_{1 x x}\right| \leq \frac{C}{|\lambda|}, \quad\left|z_{1 x x x}\right| \leq C .
$$

Although the classical theory guarantees that $z_{1}$ is an analytic function of the complex variable $\lambda$, it would be useful to give the following series expansion

$$
z_{1}(x, \lambda)=2 \sum_{n=0}^{+\infty} \frac{x^{2 n+1}}{(2 n+1) !} \sum_{k=0}^{\left[\frac{n-1}{2}\right]} C_{n}^{k}(\gamma \lambda)^{2 n-4 k-2}\left(\gamma^{4} \lambda^{4}-4 \lambda^{2}\right)^{k}
$$

which shows clearly that $z_{1}$ is an analytic function of the complex variable $\lambda$ in the whole complex plan.

Step 2. Estimates of the solution $y_{1}$. We will serve the estimates (3.11) to provide similar estimates on the solution $y_{1}$. This will be done by using Gronwall's inequality.

Proposition 3.2. - Let $-2 \beta / \gamma^{2} \leq \operatorname{Re} \lambda \leq 0$. Then the solution $y_{1}$ of the damped initial value problem (3.1) satisfies the estimates.

$$
\left|y_{1}\right| \leq \frac{C}{|\lambda|^{2}}, \quad\left|y_{1 x}\right| \leq \frac{C}{|\lambda|^{2}}, \quad\left|y_{1 x x}\right| \leq \frac{C}{|\lambda|}
$$


Proof. - Let $y_{1}$ be the unique solution of the equation (3.1). Then using the variation of constants formula, we transform the equation (3.1) into another form

$$
y_{1}(x)=z_{1}(x)-2 \lambda \int_{0}^{x} z_{1 s}(x-s)\left(a y_{1 s}\right)(s) d s
$$

It follows that

$$
\begin{aligned}
& y_{1 x}(x)=z_{1 x}(x)-2 \lambda \int_{0}^{x} z_{1 s x}(x-s)\left(a y_{1 s}\right)(s) d s, \\
& y_{1 x x}(x)=z_{1 x x}(x)-2 \lambda \int_{0}^{x} z_{1 s x x}(x-s)\left(a y_{1 s}\right)(s) d s .
\end{aligned}
$$

Using (3.11) into (3.15) gives

$$
\left|y_{1 x}(x)\right| \leq \frac{C}{|\lambda|^{2}}+\frac{2 \beta C}{|\lambda|} \int_{0}^{x}\left|y_{1 s}(s)\right| d s .
$$

Applying Gronwall's inequality, we obtain that

$$
\left|y_{1 x}(x)\right| \leq \frac{C}{|\lambda|^{2}}
$$

Inserting (3.11) and (3.17) into (3.14) gives

$$
\left|y_{1}(x)\right|=\left|z_{1}(x)\right|+2|\lambda| \int_{0}^{x}\left|z_{1 s}(x-s) \|\left(a y_{1 s}\right)(s)\right| d s \leq \frac{C}{|\lambda|^{2}} .
$$

Similarly inserting (3.11) and (3.17) into (3.16) gives

$$
\left|y_{1 x x}(x)\right|=\left|z_{1 x x}(x)\right|+2|\lambda| \int_{0}^{x}\left|z_{1 s x x}(x-s)\right|\left|\left(a y_{1 s}\right)(s)\right| d s \leq \frac{C}{|\lambda|} .
$$

The proof is thus complete.

Step 3. Approximation of the solution $y_{1}$. A natural idea is to use the "characteristic" equation involving the variable coefficient $a$ :

$$
\lambda^{2}-\left(\gamma^{2}+2 a\right) \lambda^{2} \tau^{2}+\tau^{4}=0
$$

Formlly we obtain

$$
\tau_{1,2}=\sqrt{\frac{\left(\gamma^{2}+2 a\right) \lambda^{2} \pm \sqrt{\left(\gamma^{2}+2 a\right)^{2} \lambda^{4}-4 \lambda^{2}}}{2}} .
$$

A straightforward computation gives

$$
\tau_{1}=\gamma \lambda+\frac{a}{\gamma}+O\left(\frac{1}{|\lambda|}\right), \quad \tau_{2}=\frac{1}{\gamma}+O\left(\frac{1}{|\lambda|^{2}}\right) .
$$


Neglecting the terms of high order, we see that the approximate elementary solutions of (3.1) should be

$$
\sinh \theta(x), \quad \cosh \theta(x), \quad \sinh \frac{x}{\gamma}, \quad \cosh \frac{x}{\gamma}
$$

where we have put

$$
\theta(x)=\gamma \lambda x+\frac{1}{\gamma} \int_{0}^{x} a(s) d s .
$$

Then indeed, taking into account the initial conditions

$$
v_{1}(0)=v_{1 x}(0)=v_{1 x x}(0)=0, \quad v_{1 x x x}(0)=1,
$$

we find that

$$
\begin{aligned}
v_{1}(x)=\frac{\gamma^{4}}{\Lambda}\{ & \left(1-\left(\gamma^{2} \lambda+a(0)\right)^{2}\right)\left(\sinh \theta(x)-\left(\gamma^{2} \lambda+a(0)\right) \sinh \frac{x}{\gamma}\right) \\
& \left.+\gamma a^{\prime}(0)\left(\cosh \theta(x)-\cosh \frac{x}{\gamma}\right)\right\}
\end{aligned}
$$

where we have put

$$
\begin{gathered}
\Lambda=3 a^{\prime}(0)^{2}\left(\gamma^{2} \lambda+a(0)\right) \\
-\frac{1}{\gamma}\left(1-\left(\gamma^{2} \lambda+a(0)\right)^{2}\right)\left(\left(\gamma^{2} \lambda+a(0)\right)-\left(\gamma^{2} \lambda+a(0)\right)^{3}-\gamma^{2} a^{\prime \prime}(0)\right) .
\end{gathered}
$$

Once again using the explicit expressions (3.23)-(3.24), a straightforward computation gives the following bounds

$$
\left|v_{1}\right| \leq \frac{C}{|\lambda|^{2}}, \quad\left|v_{1 x}\right| \leq \frac{C}{|\lambda|^{2}}, \quad\left|v_{1 x x}\right| \leq \frac{C}{|\lambda|} .
$$

The following result justifies that $v_{1}$ is indeed a good approximation of $y_{1}$.

Proposition 3.3. - Let $-2 \beta / \gamma^{2} \leq \operatorname{Re} \lambda \leq 0$. Assume that $a \in W^{3,1}(0,1)$. Then the following estimates hold.

$$
\left|y_{1}-v_{1}\right| \leq \frac{C}{|\lambda|^{3}}, \quad\left|y_{1 x}-v_{1 x}\right| \leq \frac{C}{|\lambda|^{3}}, \quad\left|y_{1 x x}-v_{1 x x}\right| \leq \frac{C}{|\lambda|^{2}} .
$$

Proof. - Putting

$$
L \phi=\lambda^{2} \phi-\gamma^{2} \lambda^{2} \phi_{x x}+\phi_{x x x x}-2 \lambda\left(a \phi_{x}\right)_{x},
$$

Then a straightforward computation gives

$$
\begin{aligned}
& L\left(\sinh \frac{x}{\gamma}\right)=\frac{1}{\gamma^{4}} \sinh \frac{x}{\gamma}-\frac{2 \lambda}{\gamma}\left(\frac{a}{\gamma} \sinh \frac{x}{\gamma}+a_{x} \cosh \frac{x}{\gamma}\right) \\
& L(\sinh \theta(x))=\left\{3 a_{x} \gamma \lambda^{2}+\frac{2 a a_{x}}{\gamma} \lambda+\frac{a^{2} a_{x x x}}{\gamma^{3}}\right\} \cosh \theta(x) \\
& +\left\{\left(1+a^{2}\right) \lambda^{2}+\left(4 a_{x x}+2 \frac{a^{3}}{\gamma^{2}}\right) \lambda+\frac{a^{4}}{\gamma^{4}}+\frac{9 a_{x}^{2}}{\gamma^{2}}+\frac{4 a a_{x}}{\gamma^{2}}\right\} \sinh \theta(x) .
\end{aligned}
$$


This together with (3.24) implies :

$$
\left|L v_{1}\right| \leq \frac{f_{1}}{|\lambda|}
$$

where $f_{1} \in L^{1}(0,1)$ depending only on $a$. Next writting

$$
\left\{\begin{array}{l}
\lambda^{2} v_{1}-\gamma^{2} \lambda^{2} v_{1 x x}+v_{1 x x x x}-2 \lambda\left(a v_{1 x}\right)_{x}=L v_{1}, \\
v_{1}(0)=v_{1 x}(0)=v_{1 x x}(0)=0, \quad v_{1 x x x}(0)=1 .
\end{array}\right.
$$

from the variation of constants formula, it follows that

$$
v_{1}(x)=y_{1}(x)+\int_{0}^{x} y_{1}(x-s) L v_{1}(s) d s .
$$

Then indeed using the estimates (3.13) and (3.30), we get

$$
\begin{aligned}
& \left|v_{1}(x)-y_{1}(x)\right| \leq \int_{0}^{x}\left|y_{1}(x-s)\right|\left|L v_{1}(s)\right| d s \leq \frac{C}{|\lambda|^{3}}, \\
& \left|v_{1 x}(x)-y_{1 x}(x)\right| \leq \int_{0}^{x}\left|y_{1 x}(x-s)\right|\left|L v_{1}(s)\right| d s \leq \frac{C}{|\lambda|^{3}}, \\
& \left|v_{1 x x}(x)-y_{1 x x}(x)\right| \leq \int_{0}^{x}\left|y_{1 x x}(x-s)\right|\left|L v_{1}(s)\right| d s \leq \frac{C}{|\lambda|^{2}} .
\end{aligned}
$$

Step 4. Approximation of the solution $y_{2}$. Following the same idea, we construct the approximate solution $v_{2}$ of the damped initial value problem (3.2) as follows

$$
\begin{aligned}
& v_{2}(x)=\frac{\gamma^{4}}{\Lambda}\left\{3 a^{\prime}(0)\left(\gamma^{2} \lambda+a(0)\right)\left(\sinh \theta(x)-\left(\gamma^{2} \lambda+a(0)\right) \sinh \frac{x}{\gamma}\right)\right. \\
& \left.+\frac{1}{\gamma}\left(\left(\gamma^{2} \lambda+a(0)\right)-\left(\gamma^{2} \lambda+a(0)\right)^{3}-\gamma a^{\prime \prime}(0)\right)\left(\cosh \theta-\cosh \frac{1}{\gamma}\right)\right\} .
\end{aligned}
$$

Then a straightforward computation gives

$$
\left|v_{2}\right| \leq \frac{C}{|\lambda|^{2}}, \quad\left|v_{2 x}\right| \leq \frac{C}{|\lambda|}, \quad\left|v_{2 x x}\right| \leq C .
$$

Proposition 3.4. - Let $-2 \beta / \gamma^{2} \leq$ Re $\lambda \leq 0$. Assume that $a \in W^{3,1}(0,1)$. Then the following estimates hold.

$$
\left|y_{2}-v_{2}\right| \leq \frac{C}{|\lambda|^{2}}, \quad\left|y_{2 x}-v_{2 x}\right| \leq \frac{C}{|\lambda|^{2}}, \quad\left|y_{2 x x}-v_{2 x x}\right| \leq \frac{C}{|\lambda|} .
$$

Proof. - First using (3.24), (3.28), (3.29) and (3.36), we find easily

$$
\left|L v_{2}\right| \leq f_{2}
$$


where $f_{2} \in L^{1}(0,1)$ depending only on $a$. Writting

$$
\left\{\begin{array}{l}
\lambda^{2} v_{2}-\gamma^{2} \lambda^{2} v_{2 x x}+v_{2 x x x x}-2 \lambda\left(a v_{2 x}\right)_{x}=L v_{2}, \\
v_{2}(0)=v_{2 x}(0)=v_{2 x x x}(0)=0, \quad v_{x x}(0)=1,
\end{array}\right.
$$

from the variation of constants formula, it follows that

$$
v_{2}(x)=y_{2}(x)+\int_{0}^{x} y_{1}(x-s) L v_{2}(s) d s .
$$

From (3.13) and (3.39), it follows that

$$
\begin{aligned}
& \left|v_{2}(x)-y_{2}(x)\right| \leq \int_{0}^{x}\left|y_{1}(x-s)\right|\left|L v_{2}(s)\right| d s \leq \frac{C}{|\lambda|^{2}}, \\
& \left|v_{2 x}(x)-y_{2 x}(x)\right| \leq \int_{0}^{x}\left|y_{1 x}(x-s)\right|\left|L v_{2}(s)\right| d s \leq \frac{C}{|\lambda|^{2}}, \\
& \left|v_{2 x x}(x)-y_{2 x x}(x)\right| \leq \int_{0}^{x}\left|y_{1 x x}(x-s)\right|\left|L v_{2}(s)\right| d s \leq \frac{C}{|\lambda|} .
\end{aligned}
$$

In particular, combining (3.37) and (3.38) we obtain the following bounds on $y_{2}$.

$$
\left|y_{2}\right| \leq \frac{C}{|\lambda|^{2}}, \quad\left|y_{2 x}\right| \leq \frac{C}{|\lambda|}, \quad\left|y_{2 x x}\right| \leq C .
$$

The proof is complete.

Step 5. Approximation of the function $Y(x, \lambda)$. Now we are able to give the approximation of the function $Y(x, \lambda)$.

Theorem 3.5. - Let $-2 \beta / \gamma^{2} \leq$ Re $\lambda \leq 0$. Assume that $a \in W^{3,1}(0,1)$. Then the following estimates hold.

$$
\begin{aligned}
& \left|Y_{x}(x, \lambda)-\frac{\sinh \gamma^{-1}}{\gamma^{2} \lambda^{3}} \sinh \theta(x, \lambda)\right| \leq \frac{C}{|\lambda|^{4}}, \\
& \left|Y_{x x}(x, \lambda)-\frac{\sinh \gamma^{-1}}{\gamma \lambda^{2}} \cosh \theta(x, \lambda)\right| \leq \frac{C}{|\lambda|^{3}} .
\end{aligned}
$$

Proof. - We first define the natural approximation of $Y(x, \lambda)$ by putting

$$
V(x, \lambda)=v_{1}(1, \lambda) v_{2}(x, \lambda)-v_{2}(1, \lambda) v_{1}(x, \lambda) .
$$

Then using the estimates (3.13), (3.25), (3.26), (3.37), (3.38) and (3.44), a straightforward computation shows

$$
\begin{aligned}
& \leq\left|y_{1}(1, \lambda)\right|\left|y_{2 x}(x, \lambda)-v_{2 x}(x, \lambda)\right|+\left|y_{1}(1, \lambda)-v_{1}(1, \lambda)\right|\left|y_{2 x}(x, \lambda)\right| \\
& +\left|y_{2}(1, \lambda)\right|\left|y_{1 x}(x, \lambda)-v_{1 x}(x, \lambda)\right|+\left|y_{2}(1, \lambda)-v_{2}(1, \lambda)\right|\left|y_{1 x}(x, \lambda)\right| \leq \frac{C}{|\lambda|^{4}}, \\
& \qquad\left|Y_{x x}(x, \lambda)-V_{x x}(x, \lambda)\right| \\
& \leq\left|y_{1}(1, \lambda)\right|\left|y_{2 x x}(x, \lambda)-v_{2 x x}(x, \lambda)\right|+\left|y_{1}(1, \lambda)-v_{1}(1, \lambda)\right|\left|y_{2 x x}(x, \lambda)\right| \\
& +\left|y_{2}(1, \lambda)\right|\left|y_{1 x x}(x, \lambda)-v_{1 x x}(x, \lambda)\right|+\left|y_{2}(1, \lambda)-v_{2}(1, \lambda)\right|\left|y_{1 x x}(x, \lambda)\right| \leq \frac{C}{|\lambda|^{3}} .
\end{aligned}
$$


On the other hand, from the explicit expressions (3.23), (3.24), (3.36) and (3.47) we find easily

$$
\begin{aligned}
& V_{x}(x, \lambda)=\frac{\sinh \gamma^{-1}}{\lambda^{3} \gamma^{2}} \sinh \theta(x)+O\left(\frac{1}{|\lambda|^{4}}\right), \\
& V_{x x}(x, \lambda)=\frac{\sinh \gamma^{-1}}{\lambda^{2} \gamma} \cosh \theta(x)+O\left(\frac{1}{|\lambda|^{3}}\right) .
\end{aligned}
$$

Then indeed inserting (3.50)-(3.51) into (3.48)-(3.49) gives the estimates (3.45)(3.46). The proof is thus complete.

Now for any integer $n \geq 0$, we define the following sets

$$
\begin{aligned}
& C_{N}=\{-2 \beta / \gamma \leq \operatorname{Re} \gamma \lambda \leq 0,-n \pi \leq \operatorname{Im} \gamma \lambda \leq n \pi\} \\
& G_{ \pm n}=\{-2 \beta / \gamma \leq \operatorname{Re} \gamma \lambda \leq 0, \quad \pm(n-1 / 2) \pi \leq \operatorname{Im} \gamma \lambda \leq \pm(n+1 / 2) \pi\} \\
& \Gamma_{ \pm n}=\left\{|\gamma \lambda+\bar{a} \mp i n \pi| \leq C \gamma^{3} / n \sinh \gamma^{-1}\right\}
\end{aligned}
$$

where we have put

$$
\bar{a}=\frac{1}{\gamma} \int_{0}^{1} a(x) d x
$$

Proposition 3.6. - There exists an integer $N$ such that for any $n>N$ the following estimate holds

$$
|\sinh (\gamma \lambda+\bar{a})|>\frac{C \gamma^{2}}{|\lambda| \sinh \gamma^{-1}}, \quad \lambda \in \partial \Gamma_{ \pm n} \cup \partial G_{ \pm n} .
$$

Proof. - First let $\lambda=u+i v \in \partial G_{ \pm n}$, then we have

$$
|\sinh (\gamma \lambda+\bar{a})|^{2}=\sin ^{2}(\gamma v)+\sinh ^{2}(\gamma u+\bar{a}) .
$$

If $u=0$ or $\gamma u=-2 \beta / \gamma$, then we have

$$
|\sinh (\gamma \lambda+\bar{a})| \geq|\sinh \bar{a}| .
$$

If $\gamma v=(n \pm 1 / 2) \pi$, then

$$
|\sinh (\gamma \lambda+\bar{a})| \geq 1
$$

Setting $\mu=\min \{1, \sinh \bar{a}\}$, then we obtain

$$
|\sinh (\gamma \lambda+\bar{a})| \geq \mu, \quad \forall \lambda \in \partial G_{ \pm n} .
$$

But $|\gamma \lambda| \geq(n-1 / 2) \pi$ for $\lambda \in \partial G_{ \pm n}$ then we obtain (3.56) provided that

$$
n>\frac{C \gamma^{3}}{\mu \pi \sinh \gamma^{-1}}+\frac{1}{2}
$$


Now let $\lambda \in \partial \Gamma_{ \pm n}$. Writting

$$
\gamma \lambda+\bar{a}= \pm i n \pi+\rho_{n} e^{i \theta}, \quad \rho_{n}=\frac{C \gamma^{3}}{n \sinh \gamma^{-1}}, \quad \theta \in[0,2 \pi[,
$$

we deduce that

$$
|\sinh (\gamma \lambda+\bar{a})|=\sqrt{\sinh ^{2}\left(\rho_{n} \cos \theta\right)+\sin ^{2}\left(\rho_{n} \sin \theta\right)} \geq \frac{2}{\pi} \rho_{n}=\frac{2 C \gamma^{3}}{n \pi \sinh \gamma^{-1}} .
$$

Since $|\gamma \lambda| \geq n \pi-\bar{a}-\gamma^{3} C / \sinh \gamma^{-1}$, then we obtain (3.56) provided that

$$
n>\frac{2\left(\bar{a} \sinh \gamma^{-1}+\gamma^{2} C\right)}{\pi \sinh \gamma^{-1}}
$$

Finally we complete the proof by choosing

$$
N=\max \left(\frac{\gamma^{3} C}{\mu \pi \sinh \gamma^{-1}}+\frac{1}{2}, \quad \frac{2\left(\bar{a} \sinh \gamma^{-1}+\gamma^{3} C\right)}{\pi \sinh \gamma^{-1}}\right)
$$

Theorem 3.7. - Let $a \in W^{3,1}(0,1)$. Then A has one simple eigenvalue in each neighborhood $\Gamma_{n}$ for $n>N$, and a finite number of eigenvalues in $C_{N}$. This exhauts the spectrum of $A$.

Proof. - From Proposition 2.1, we know that the spectrum $\sigma(A)$ is contained in the strip $-2 \beta / \gamma^{2} \leq \operatorname{Re} \lambda \leq 0$. Let $n>N$, then using (3.45) and (3.56) we have

$$
\left|Y_{x}(1, \lambda)-\sinh \gamma^{-1} \frac{\sinh (\gamma \lambda+\bar{a})}{\gamma^{2} \lambda^{3}}\right| \leq \frac{C}{|\lambda|^{4}}<\left|\sinh \gamma^{-1} \frac{\sinh (\gamma \lambda+\bar{a})}{\gamma^{2} \lambda^{3}}\right|
$$

for any $\lambda \in \partial \Gamma_{ \pm n} \cup \partial G_{ \pm n}$. Via Rouché's theorem we know that $Y_{x}(1, \lambda)$ possesses one simple zero $\lambda_{n}$ in each neighborhood $\Gamma_{ \pm n}$, and this exhauts the zeros of $Y_{x}(1, \lambda)$ in the region $G_{ \pm n}$. In particular, we have

$$
\left|\gamma \lambda_{n}+\bar{a} \mp i n \pi\right| \leq \frac{C \gamma^{3}}{n \sinh \gamma^{-1}} .
$$

Since the spectrum $\sigma(A)$ is discrete and $C_{N}$ is compact, then there exists a finite number of eigenvalues in $C_{N}$. The proof is complete.

Theorem 3.8. - Let $a \in W^{3,1}(0,1)$. Putting

$$
\begin{aligned}
& y_{n}(x)=\frac{\lambda_{n}^{3} \gamma^{2}}{\sinh \gamma^{-1}} Y\left(x, \lambda_{n}\right), \quad|n|>N, \\
& \xi(x)=\frac{1}{\gamma} \int_{0}^{x} a(s) d s-\frac{x}{\gamma} \int_{0}^{1} a(s) d s,
\end{aligned}
$$

then we have

$$
\begin{aligned}
& \left|\gamma \lambda_{n} y_{n x}(x)-\sinh (\xi(x)+i n \pi x)\right|=O\left(\frac{1}{n}\right), \\
& \left|y_{n x x}(x)-\cosh (\xi(x)+i n \pi x)\right|=O\left(\frac{1}{n}\right) .
\end{aligned}
$$


Proof. - From (3.22) and (3.59), we obtain

$$
\theta\left(x, \lambda_{n}\right)=\frac{1}{\gamma} \int_{0}^{x} a(s) d s-\frac{x}{\gamma} \int_{0}^{1} a(s) d s x+i n \pi x+O\left(\frac{1}{n}\right) .
$$

Inserting (3.64) into (3.45)-(3.46) gives (3.62)-(3.63). The proof is complete.

\section{System of root vectors}

Let $\lambda_{n}$ be an eigenvalue of $A$. Then from Theorem 3.7 we know that the algebraic multiplicity $m_{n}=1$ for $|n|>N$. We will numerote the eigenvalues $\lambda_{n}$ of high frequencies $(|n|>N)$ following the asymptotic form (3.59). For clarity, we denote by $\widetilde{\lambda}_{k}$ the eigenvalues of low frequencies $(0 \leq k \leq K)$ with algebraic multiplicity $m_{k} \geq 1$. Then we can write the spectrum of $A$ into the following form :

$$
\sigma(A)=\left\{\lambda_{n}, \quad|n|>N\right\} \cup\left\{\widetilde{\lambda}_{k}, \quad 0 \leq k \leq K\right\} .
$$

Correspondingly, we denote by $\phi_{n}=\left(y_{n}, \lambda_{n} y_{n}\right)$ the eigenvector associated to the eigenvaue $\lambda_{n}$ of high frequency, and by $\left\{\widetilde{\phi}_{k, j}\right\}_{0}^{m_{k}}$ the Jordan chain of root vectors associated to the eigenvalue $\widetilde{\lambda}_{k}$ of low frequency. Thus we obtain a system of root vectors of $A$ :

$$
\left\{\phi_{n}: \quad|n|>N\right\} \cup\left\{\widetilde{\phi}_{k, j}, \quad 0 \leq j \leq m_{k}, \quad 0 \leq k \leq K\right\} .
$$

Denoting by $\mathcal{L}$ the subspace

$$
\mathcal{L}=\left(\begin{array}{c}
(f, g) \in L^{2}(0,1) \times L^{2}(0,1) \\
\int_{0}^{1} f(x) d x=\int_{0}^{1} x f(x) d x=\int_{0}^{1} g(x) d x=0
\end{array}\right)
$$

we define the linear bonuded operator $T$ by setting

$$
T(y, z)=\left(y_{x x}, z_{x}\right), \quad \forall(y, z) \in H_{0}^{2}(0,1) \times H_{0}^{1}(0,1) .
$$

Proposition 4.1. - The operator $T$ definerd in (4.3)-(4.4) is an isomorphism from $H_{0}^{2}(0,1) \times H_{0}^{1}(0,1)$ onto $\mathcal{L}$.

Proof. - It's easy to verify that $T$ is bounded from $H_{0}^{2}(0,1) \times H_{0}^{1}(0,1)$ into $\mathcal{L}$. On the other hand, for any $(f, g) \in \mathcal{L}$ there exist a unique pair $(y, z)$ given by

$$
y=\int_{0}^{x}(x-s) f(s) d s \in H_{0}^{2}(0,1), \quad z=\int_{0}^{x} g(s) d s \in H_{0}^{1}(0,1)
$$

such that $T(y, z)=(f, g)$. Therefore by Banach's well-known theorem, we deduce that $T$ is an isomorphism from $H_{0}^{2}(0,1) \times H_{0}^{1}(0,1)$ onto $\mathcal{L}$. The proof is complete.

Now for any $\xi \in L^{\infty}(0,1)$, we define the system

$$
\Phi_{n}=\left(\cosh (\xi(x)+i n \pi), \gamma^{-1} \sinh (\xi(x)+i n \pi)\right), \quad n \in \mathcal{Z} .
$$


We will prove later that the system (4.5) is a Riesz basis in $L^{2}(0,1) \times L^{2}(0,1)$. Then we see that the estimates (3.62)-(3.63) mean exactly that the system $\left\{T \phi_{n}\right\}_{|n|>N}$ is quadratically close to the sub-system $\left\{\Phi_{n}\right\}_{|n|>N}$ in the space $L^{2}(0,1) \times L^{2}(0,1)$. On the other hand, the number of eigenvalues $\widetilde{\lambda}_{k}$ of low frequencies : $\sum_{k=0}^{K} m_{k}$, including algebraic multiplicities is a priori less than $2 N+1$. This means that there is no one to one mapping between the complementaries $\left\{T \widetilde{\phi}_{k, j}, \quad 0 \leq j \leq m_{k}, \quad 0 \leq k \leq K\right\}$ and $\left\{\Phi_{n}\right\}_{|n| \leq N}$. Therefore Bari's classical theorem can't be applied directly to the system of root vectors (4.2). Hence we need to establish the following result.

Theorem 4.2. - Let $\left\{\psi_{n}\right\}_{0}^{\infty}$ be a Riesz basis in the Hilbert space $X$, and let $\left\{g_{n}\right\}_{n_{0}}^{\infty}$ be a $\omega$-linearly independent system. Assume that

$$
\sum_{n=n_{0}}^{\infty}\left\|\psi_{n}-g_{n}\right\|_{X}^{2}<+\infty
$$

Then $\left\{g_{n}\right\}_{n_{0}}^{\infty}$ is a Riesz basis in the subspace $X_{0}$ spanned by itself in $X$.

Proof. - We will adopte the same method as that one used in Gohberg-Krein [9, Thm. VI.2.3].

Since $\left\{\psi_{n}\right\}_{0}^{\infty}$ is a Riesz basis in the Hilbert space $X$, then there exist a linear bounded invertible operator $S$, and an orthonormal basis $\left\{\Psi_{n}\right\}_{0}^{\infty}$ in the space $X$ such that (Gohberg-Krein [9, Thm. VI.2.1])

$$
S \Psi_{n}=\psi_{n}, \quad n=0,1,2, \cdots .
$$

Defining the operator $T$ by setting

$$
T\left(\sum_{n=0}^{\infty} c_{n} \Psi_{n}\right)=\sum_{n=0}^{n_{0}-1} c_{n} \psi_{n}+\sum_{n=n_{0}}^{\infty} c_{n}\left(\psi_{n}-g_{n}\right) \quad \text { for } \quad \sum_{n=0}^{\infty}\left|c_{n}\right|^{2}<+\infty,
$$

then from (4.6) comes

$$
\sum_{n=0}^{\infty}\left\|T \Psi_{n}\right\|^{2}=\sum_{n=0}^{n_{0}-1}\left\|\psi_{n}\right\|^{2}+\sum_{n=n_{0}}^{\infty}\left\|\psi_{n}-g_{n}\right\|^{2}<+\infty
$$

This proves that $T$ is an Hilbert-Schmidt operator $X$.

On the other hand, from (4.7)-(4.8) we have

$$
(S-T)\left(\sum_{n=0}^{\infty} c_{n} \Psi_{n}\right)=\sum_{n=n_{0}}^{\infty} c_{n} g_{n} \quad \text { for } \quad \sum_{n=0}^{\infty}\left|c_{n}\right|^{2}<+\infty .
$$

Then it follows that

$$
R(S-T) \subset X_{0}
$$

But $R(S-T)$ is closed and contains all the finite linear combinations of the system $\left\{g_{n}\right\}_{n_{0}}^{\infty}$, this gives that

$$
X_{0} \subset R(S-T)
$$


which together with (4.10) imply that $R(S-T)=X_{0}$. Hence we have proved that $S-T$ is a linear bounded operator from the subspace $E$ spanned by the subsystem $\left\{\Psi_{n}\right\}_{n_{0}}^{\infty}$ onto the subspace $X_{0}$ spanned by the subsystem $\left\{g_{n}\right\}_{n_{0}}^{\infty}$

Now let

$$
\Psi=\sum_{n=n_{0}}^{\infty} c_{n} \Psi_{n} \in E \quad \text { such that } \quad(S-T) \Psi=0 .
$$

Then from (4.9) it follows that

$$
\sum_{n=n_{0}}^{\infty} c_{n} g_{n}=0 \quad \text { for } \quad \sum_{n=n_{0}}^{\infty}\left|c_{n}\right|^{2}<+\infty
$$

Since the system $\left\{g_{n}\right\}_{n_{0}}^{\infty}$ is $\omega$-linearly independent, it follows that $c_{n}=0$ for all $n \geq n_{0}$. From Banach's well-known theorem, we deduce that $S-T$ is an isomorphism from $E$ onto $X_{0}$. Bearing in mind the following obvious equalities

$$
(S-T) \Psi_{n}=g_{n}, \quad \forall n \geq n_{0},
$$

we conclude that the system $\left\{g_{n}\right\}_{n_{0}}^{\infty}$ is a Riesz basis in the subspace $X_{0}$. This achieves the proof.

Now given any $(f, g) \in H_{0}^{2}(0,1) \times H_{0}^{1}(0,1)$, we solve the equation $A(y, z)=(f, g)$. Then a direct computation gives that

$$
y=G_{4} D f-G_{4}\left(I-\gamma^{2} \partial_{x x}\right) g, \quad z=f
$$

where $D=2 \partial_{x}\left(a \partial_{x}\right)$ is a linear bounded operator from $H_{0}^{1}(0,1)$ into $H^{-1}(0,1)$, and $G_{4}=\left(\partial_{x x x x}\right)^{-1}$ is an isomorphism from $H^{-2}(0,1)$ onto $H_{0}^{2}(0,1)$ such that

$$
\int_{0}^{1}\left(G_{4} y\right)_{x x} \bar{\phi}_{x x} d x=\langle y, \phi\rangle_{H^{-2}(0,1) \times H_{0}^{2}(0,1)}, \quad \forall \phi \in H_{0}^{2}(0,1) .
$$

Then from (4.13) comes the following expression

$$
i A^{-1}=i\left(\begin{array}{cc}
0 & -G_{4}\left(I-\gamma^{2} \partial_{x x}\right) \\
I & 0
\end{array}\right)+i\left(\begin{array}{cc}
G_{4} D & 0 \\
0 & 0
\end{array}\right) .
$$

We next define the linear bounded operators

$$
L=i\left(\begin{array}{cc}
0, & -G_{4}\left(I-\gamma \partial_{x x}\right) \\
I, & 0
\end{array}\right), \quad S=\left(\begin{array}{cc}
0, & 0 \\
-G_{2} D, & 0
\end{array}\right)
$$

where $G_{2}=\left(I-\gamma^{2} \partial_{x x}\right)^{-1}$ is an isomorphism from $H^{-1}(0,1)$ onto $H_{0}^{1}(0,1)$ such that

$$
\int_{0}^{1}\left(G_{2} y \bar{\phi}+\gamma^{2}\left(G_{2} y\right)_{x} \bar{\phi}_{x}\right) d x=\langle y, \phi\rangle_{H^{-1}(0,1) \times H_{0}^{1}(0,1)}, \quad \forall \phi \in H_{0}^{1}(0,1) .
$$


Then we can write (4.15) into the following form

$$
i A^{-1}=L(I+S) .
$$

Proposition 4.3. - The system (4.2) of root vectors of $A$ is complete in the energy space $H=H_{0}^{2}(0,1) \times H_{0}^{1}(0,1)$.

Proof. - Since $G_{2} D$ is continuous in $H_{0}^{1}(0,1)$, then from the compact embedding $H_{0}^{2}(0,1) \hookrightarrow H_{0}^{1}(0,1)$ we observe that $S$ is compact in the energy space $H_{0}^{2}(0,1) \times H_{0}^{1}(0,1)$. Similarly from the compact embedding $H^{-1}(0,1) \hookrightarrow H^{-2}(0,1)$, we deduce that $G_{4}\left(I-\gamma^{2} \partial_{x x}\right)$ is compact from $H_{0}^{1}(0,1)$ into $H_{0}^{2}(0,1)$. This implies that $L$ is also compact in the energy space $H_{0}^{2}(0,1) \times H_{0}^{1}(0,1)$. Moreover using (4.17), an elementary computation gives that

$$
\langle L(y, z),(f, g)\rangle_{H}=\int_{0}^{1}\left(z(\overline{i f})+\gamma^{2} z_{x}(\overline{i f})_{x}-y(\overline{i g})-\gamma^{2} y_{x}(\overline{i g})_{x}\right) d x
$$

for all $(y, z),(f, g) \in H_{0}^{2}(0,1) \times H_{0}^{1}(0,1)$. This implies that $L$ is selfadjoint in the energy space $H=H_{0}^{2}(0,1) \times H_{0}^{1}(0,1)$.

On the other hand, in the case $a=0$ we have $L=i A^{-1}$. Then using (3.59) we obtain the following asypmtotic expansion of the eigenvalues of $L$

$$
\lambda_{n}(L)=\frac{i}{\lambda_{n}(A)}=\frac{i \gamma}{i n \pi+O(1 / n)}=\frac{\gamma}{n \pi}+O\left(\frac{1}{n^{3}}\right) .
$$

Since $L$ is compact and selfadjoint, then its $s$-numbers are given by (GohbergKrein $[9$, p. 27]).

$$
s_{n}(L)=\left|\lambda_{n}(L)\right|, \quad \forall n \geq 0 .
$$

Therefore $L$ is of finite order : $p(L)=1$ (Gohberg-Krein [9, p. 256]). Then we conclude that the system of root vectors of $i A^{-1}$ is complete (Gohberg-Krein [9, Thm. VI. 8.1). This achieves the proof.

Proposition 4.4. - The system (4.2) of root vectors of $A$ is w-linearly independent in the energy space $H_{0}^{2}(0,1) \times H_{0}^{1}(0,1)$.

Proof. - Since $L$ is selfadjoint, from (4.16) we obtain the imaginary component of $i A^{-1}$

$$
\operatorname{Im}\left(i A^{-1}\right)=\operatorname{Im} i\left(\begin{array}{cc}
G_{4} D & 0 \\
0 & 0
\end{array}\right)
$$

Since $G_{4} D$ is compact in $H_{0}^{2}(0,1)$, so is $\operatorname{Im}\left(i A^{-1}\right)$ in the energy space $H_{0}^{2}(0,1) \times H_{0}^{1}(0,1)$. 
Now let $\lambda_{n}$ be an eigenvalue of $A$. Since $A$ has no pur imaginary eigenvalue, then $i A^{-1}$ has non real eigenvalue. Therefore $i / \lambda_{n}$ is a normal eigenvalue of $i A^{-1}$ (Gohberg-Krein [9, Thm. I.5.2]). Hence for sufficiently small $r_{n}>0$, the operator

$$
P_{n}=-\frac{1}{2 \pi i} \int_{\left|\lambda-i / \lambda_{n}\right|=r_{n}}\left(i A^{-1}-\lambda I\right)^{-1} d \lambda
$$

projects $H_{0}^{2}(0,1) \times H_{0}^{1}(0,1)$ onto the root subspace $\operatorname{Ker}\left(A-\lambda_{n} I\right)^{m_{n}}$.

Now assume that

$$
\sum_{k=1}^{K} \sum_{j=0}^{m_{k}-1} c_{k, j} \widetilde{\phi}_{k, j}+\sum_{|n|>N} c_{n} \phi_{n}=0 .
$$

Applying the projector $P_{n}$ to (4.24) gives that

$$
c_{k, j}=0, \quad 0 \leq j \leq m_{k}-1, \quad 1 \leq k \leq K ; \quad c_{n}=0, \quad|n|>N .
$$

The proof is complete.

From Proposition 4.1, we know that the operator $T$ defined in (4.3)-(4.4) is an isomorphism from $H_{0}^{2}(0,1) \times H_{0}^{1}(0,1)$ onto the subspace $\mathcal{L}$, then the following result is an immediate consequence of Propositions 4.3 and 4.4.

Corollary 4.5. - The following system:

$$
\left\{T \widetilde{\phi}_{k, j}: \quad 0 \leq j \leq m_{k}-1, \quad 0 \leq k \leq K\right\} \cup\left\{T \phi_{n}: \quad|n|>N\right\}
$$

is complete and $\omega$-linearly independent in the subspace $\mathcal{L}$.

Proposition 4.6. - For any $\xi \in L^{\infty}(0,1)$, the system (4.5) is a Riesz basis in $L^{2}(0,1) \times L^{2}(0,1)$

Proof. - A straightforward computation gives that

$$
\Phi_{n}(x)=(\cos (n \pi x), \sin (n \pi x))\left(\begin{array}{cc}
\cosh \xi(x), & \frac{1}{\gamma} \sinh \xi(x) \\
i \sinh \xi(x), & \frac{i}{\gamma} \cosh \xi(x)
\end{array}\right) .
$$

Since the transformation matrix has a bounded inverse in $L^{2}(0,1) \times L^{2}(0,1)$, and since the system $(\cos (n \pi x), \sin (n \pi x))$ is equivalent to an orthonormal basis in $L^{2}(0,1) \times L^{2}(0,1)$, it follows that the system (4.5) is a Riesz basis in $L^{2}(0,1) \times L^{2}(0,1)$. The proof is complete.

TheORem 4.7. - Assume that $a \in W^{3,1}(0,1)$. Then the system of root vectors (4.2) is a Riesz basis in $H_{0}^{2}(0,1) \times H_{0}^{1}(0,1)$.

Proof. - Since the operator $T$ defined in (4.3)-(4.4) is an isomorphism from $H_{0}^{2}(0,1) \times H_{0}^{1}(0,1)$ onto $\mathcal{L}$ (Proposition 4.1), then it is sufficient to prove that the system (4.25) is a Riesz basis in the space $\mathcal{L}$. 
But from the estimates (3.62)-(3.63), it follows that

$$
\sum_{|n|>N}\left\|T \phi_{n}-\Phi_{n}\right\|_{L^{2}(0,1) \times L^{2}(0,1)}^{2}<+\infty .
$$

On the other hand, we know that the system (4.25) is $\omega$-linearly independent (Corollary 4.5) and the system (4.5) is Riesz basis in $L^{2}(0,1) \times L^{2}(0,1)$ (Proposition 4.6), then applying Theorem 4.2 we conclude that the system (4.25) is a Riesz basis in the subspace spanned by itself in $\mathcal{L}$, therefore in the whole space $\mathcal{L}$, since it is complete in $\mathcal{L}$ (Corollary 4.5). This achieves the proof.

Theorem 4.8. - Assume that $a \in W^{3,1}(0,1)$. Then we have

$$
\omega(a)=\mu(a)
$$

The proof is analogous to that one in Cox-Zuazua [6] and Morgul et al [11], therefore we omit it here.

\section{Acknowlegments}

The author would like to thank Francis Conrad for helpful discussions and suggestions. This reseach was partially supported by the Projet Numath, INRIA Lorraine, France.

\section{References}

[1] G. D. Birkoff; R. E. Langer, The boundary problem and developements associated with a system of ordinary differential equations of first order, Proc. Amer. Acad. Arts Sci., 58 (1923), pp. 51-128.

[2] G. Chen; S.G. Krantz; D. W. Ma; C.E. Wayre; H. H. West, The Euler-Bernoulli beam equation with boundary energy dissipation, Operator Methods for Optimal Control Problems, ed. S.J. Lee, Lecture in Pure Appl. Math. Series (1987), pp. $67-96$.

[3] G. Chen; C. Delfour; A.M. Krall; G. Payre, Modelling, satabilization and control of serially connected beams, SIAM J. Control Optim. 25 (1987), pp. 526-546.

[4] G. Chen, S. A. Fulling, F. G. Narcowich and C. Qi An asymptotic average decay rate for the wave equation with variable coefficient viscous damping, SIAM J. Appl. Math. 50 (1990), pp. 1341-1347.

[5] E. Coddington, N. Levinson, Theory of ordinary differential equations, Mc. Graw Hill, 1955.

[6] S. Cox; E. Zuazua, The rate at which energy decays in a damped string, Commun. in PDE 19 (1994), pp. 213-244.

[7] S. Cox; E. Zuazua, The rate at which energy decays in a string damped at one end, Indiana Univ. Math. J., 44, pp. 507-535.

[8] P. Freitas; E. Zuazua, Stability results for wave equation with indefinite damping, J. Differential Equations, 132 (1996), pp. 338-353. 
[9] I. C. Gohberg; M.G. Krein, Introduction to the theory of linear nonselfadjoint operators, AMS, Providence, 1969.

[10] J. E. Lagnese, Boundary stabilization of thin plates, SIAM Publications, Philadelphia, 1989.

[11] O. Morgul; F. Conrad; B. Rao, On the stabilisation of a cable with a tip mass, IEEE Transactions on Automatic Control, 39 (1994), pp. 2140-2145.

[12] M. A. Naimark, Linear differential operators, Vol. I, Ungar, New York, 1967.

[13] A. Pazy, Semigroups of linear operators and applications to partial differential equations, Springer-Verlag, New York, (1983).

[14] B. Rao, A compact perturbation method for the boundary srtabilization of the Rayleigh beam equation, Appl. Math. Optim. 33 (1996), pp. 253-264.

[15] D. L. Russell, On the mathematical models for the elastic beam with frenquenceproportional damping, Control and Estimation in Distributed Parameters Systems, ed. H. T. Bank, Frontiers in Applied Mathematics, Philadelphial, (1992), pp. 125-169. 